\title{
Toward Adaptation of fNIRS Instrumentation to Airborne Environments
}

\author{
G. Adamovsky*1, J. Mackey ${ }^{2}$, A. Harrivel ${ }^{1}$, T. Hearn ${ }^{1}$, and B. Floyd ${ }^{3}$ \\ ${ }^{1}$ NASA Glenn Research Center, Cleveland Ohio, 44135 \\ ${ }^{2}$ Vantage Partners, Cleveland Ohio, 44135 \\ ${ }^{3}$ Sierra Lobo Inc., Cleveland Ohio, 44135
}

\begin{abstract}
The paper reviews potential applications of functional Near-Infrared Spectroscopy (fNIRS), a well-known medical diagnostic technique, to monitoring the cognitive state of pilots with a focus on identifying ways to adopt this technique to airborne environments. We also discuss various fNIRS techniques and the direction of technology maturation of associated hardware in view of their potential for miniaturization, maximization of data collection capabilities, and user friendliness.
\end{abstract}

\section{Introduction}

$\mathrm{T}$ he reduction of accident rates is of great importance in the face of increasing commercial aviation traffic. Even the most expert and conscientious pilots are susceptible to making errors in stressful situations. Intelligent cockpits of the future will interact with operators in ways designed to reduce error prone states and mitigate dangerous situations at the edges of human performance.

NASA's Aviation Safety Program has recognized the role of pilots and their ability to respond in critical flight situations as one of the major elements in its continuing effort to improve the safety of commercial flight. It has also established, as a part of the Vehicle System Safety Technologies (VSST) Project, a theme of technical challenges to deal with crew decision making and responses in complex situations. The crew decision making (CDM) technical area includes a broad range of technologies dealing with safe operation of piloted airspace vehicles. It has been formulated in response to safety concerns coupled with the historical trends and the forecasted changes ushered in by advances in global air transportation. CDM is aimed at developing data and technologies that enable new capabilities for pilots to better understand and respond safely to complex situations, thereby increasing the pilots' ability to avoid, detect, and recover from adverse events that could otherwise result in accidents/incidents.

One important aspect of this is the development of reliable, sensitive and operationally-relevant metrics for the state of the "human in the loop" by noninvasive, portable, safe, and inexpensive means. The overall goal is to reduce the effects of performance decrement and improve safety by informing intelligent systems of the state of the operator. Appropriate injections of mitigations and operator support by an intelligent cockpit may then be made before cognitive decision-making abilities are compromised.

Monitoring of the aviation pilot's situational awareness has become a subject of intensive studies over the past several years. ${ }^{1-4}$ In the process of these studies, such diverse techniques as eye and gaze tracking and blood oxygen level monitoring have been considered.

In this paper we will discuss issues associated with implementation in airborne environments of a spectroscopic blood oxygen level monitoring technique called functional Near-Infrared Spectroscopy (fNIRS).

\section{Functional Near-Infrared Spectroscopy (fNIRS)}

Near-Infrared Spectroscopy (NIS) is a medical tomographic technique to analyze structural properties of tissues $^{5,6}$ and functional responses of a brain ${ }^{7,8}$ by non-invasively measuring concentration changes of oxy- and deoxy-hemoglobin $\left(\mathrm{HbO}_{2}\right.$ and $\left.\mathrm{Hb}\right)$ and cytochome oxidase (CtOx). Monitoring of those parameters also helps evaluating the cognitive state of the patient, for example in tracking the development of Alzheimer's disease, and studying other brain-behavior relationships. The monitoring is usually done through utilization of two or more wavelengths of light selected such that the light propagation properties at those wavelengths are significantly

\footnotetext{
${ }^{*}$ Sr. Research Engineer, Optical Instrumentation \& NDE Branch, AIAA Associate Follow.
} 
different for oxygenated and deoxygenated tissues. In applications of NIS to detection of damaged or abnormal tissue regions, variations in the light absorption of tissue permit, for instance, an early detection of cancer.

In functional Near-Infrared Spectroscopy (fNIRS), the light propagating at each wavelength through the tissue and blood-carrying vessels exhibits different properties, depending not only on the wavelengths used but also on the properties of the blood in the vessels. Processing information on the relative changes in concentration of $\mathrm{HbO}_{2}$ and $\mathrm{Hb}$ in blood vessels at particular locations in the brain indirectly determines the state of awareness of the subject or crewmember and his or her ability to make decisions or respond to situational changes.

In general, the effectiveness of fNIRS applications to clinical analysis depends on several factors including design of electro-optic instrumentation, stability of human-machine mechanical contacts, "client" or subject cooperation, absorption and scattering of light by human tissue, availability of proper means to process and interpreted information, and other. ${ }^{9-11}$ Adaptation of fNIRS to airborne environments adds other factors specific to the aircraft. For instance, while not considered of a significant importance in clinical environments, the size and weight of electro-optical instrumentation including signal processing will be limited when placing such instrumentation in the cockpit of an aircraft. There are three basic types of fNIRS instrumentation: continuous intensity, intensity modulated (or frequency domain), and time resolved. The first type, continuous intensity, is based on evaluation of the absorption of light passing through tissue. It provides intensity measurements at different wavelengths. The light sources in this case emit at constant intensity levels. In intensity modulated instrumentation, the light that enters the tissue is modulated and three parameters of the light passing through it are measured: intensity, phase, and modulation depth. Finally, the time resolved type employs a short pulse of light which after passing through tissue is spread in time. Each of these types has advantages and disadvantages with the continuous intensity being the most commonly used. A more detailed description of the fNIRS techniques and principles of their operations can be found elsewhere. ${ }^{12-14}$ The hardware and hardware-related components of any fNIRS system can be loosely grouped as electro-optic instrumentation, headgear and fiber interfaces, and associated signal processing and software. In a particular system they are highly integrated and in many cases it is impossible to discuss one group of components without mentioning others.

Table 1 outlines major areas of hardware and software related elements of fNIRS techniques.

Table 1. Major instrumentation and software related elements of fNIRS techniques

\begin{tabular}{|l|l|l|}
\hline ELEMENTS & EVALUATION MATRIX & LIMITATIONS \\
\hline Electro-optic Instrumentation & $\begin{array}{l}\text { Signal-to-Noise Ratio } \\
\text { Small size and Low Weight } \\
\text { Low Power; Cost }\end{array}$ & $\begin{array}{l}\text { Operating wavelengths } \\
\text { Sources and detectors } \\
\text { Growing but limited vendor base }\end{array}$ \\
\hline $\begin{array}{l}\text { Human-machine interface } \\
\text { (headgear) and test subject } \\
\text { cooperation }\end{array}$ & $\begin{array}{l}\text { User friendliness and safety } \\
\text { Interface mechanical stability }\end{array}$ & $\begin{array}{l}\text { Acceptance by users / clients } \\
\text { Insufficient usage data base } \\
\text { Privacy concerns }\end{array}$ \\
\hline Software and signal processing & $\begin{array}{l}\text { Efficiency and credibility of } \\
\text { processed information }\end{array}$ & $\begin{array}{l}\text { data storage, processor speed, flight } \\
\text { automation firmware compatibility, } \\
\text { artifact removal algorithms }\end{array}$ \\
\hline
\end{tabular}

\section{A. Electro-Optic Instrumentation Element in fNIRS Systems}

The electro-optic instrumentation element is a key part of any fNIRS system. It consists of light sources emitting at particular wavelengths, photodetectors, and a system of optical fibers connecting the sources and detectors to a human head. Light from the sources reaches the head via optical fibers, gets scattered in the tissue, and a portion of the scatted light is coupled back into optical fibers and delivered to photodetectors for analysis. Studies have shown that in order to penetrate several centimeters through the tissue, the wavelength of the light should be between 600 and $900 \mathrm{~nm} \cdot{ }^{15,16}$ Furthermore, absorption of light at the shorter range of the wavelengths is significantly affected by the presence of oxygen in the human tissue. At the same time, light at wavelengths between 800 and $850 \mathrm{~nm}$ experiences absorption that practically is not affected by the level of oxygenation. ${ }^{17,18}$

The selection of sources and detectors is directly dependent on the light wavelength selected, and the configuration of the fNIRS system itself.

Design of the instrumentation element of any fNIRS system is driven by the electrical power budget and associated with it the signal-to-noise ratio because of the selection of detector type. That is especially important in aerospace environments with limited weight and size as well as power availability. A schematic diagram 
representing the fNIRS concept is shown in Fig. 1. Light emitted from a light source at one of the operating wavelengths is coupled into one or several optical fibers and delivered to a light injector placed on a human head. Through the injector, the light is sent through the skull into the brain. In the brain, the light experiences significant scattering and some absorption, with the absorption especially being dependant on both the wavelength used and the levels of both deoxy- and oxygenated hemoglobin in the blood. Overall, just a small portion of light is being collected by the light collector placed also on the head centimeters from the injector.

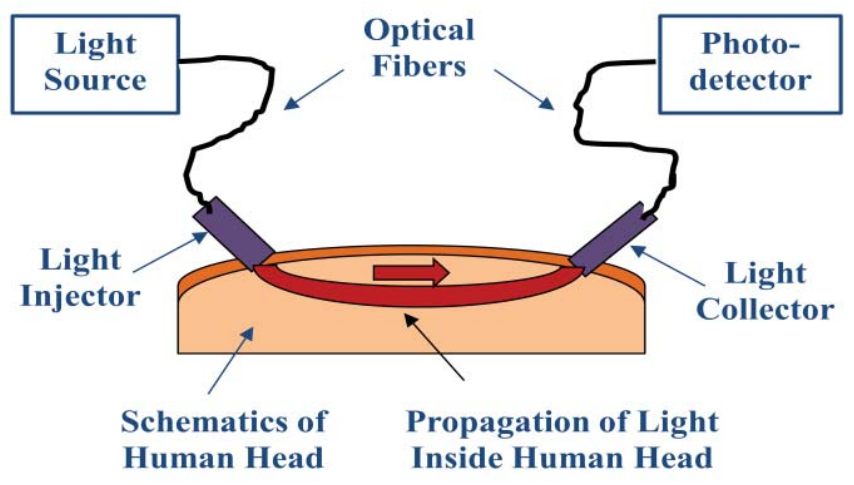

Figure 1. Schematic diagram of the fNIRS concept.

In a classical continuous intensity fNIRS configuration, the laser sources emit continuously. However, in practice the sources are intensity or pulse-modulated at low frequencies to increase the signal-to-noise ratio and to provide multiplexing and demultiplexing. ${ }^{19,20}$ While the continuous intensity configuration of fNIRS is not suited for absolute characteristics of the tissue optical properties, ${ }^{21}$ it is the most common configuration used in brain imaging. ${ }^{22}$

In fNIRS systems employing high frequency intensity modulation, the modulation is achieved by applying a sinusoidally varying current to a laser diode junction that is under a certain constant voltage bias. ${ }^{23-25}$ Both the constant bias and sinusoidally varying current generate optical radiation with corresponding DC and AC components. In the intensity modulation fNIRS both parts of radiation can be measured and their dimensionless ratio used as an additional parameter for data evaluation ${ }^{26}$. Furthermore, an improved signal-to-noise ratio has been reported. ${ }^{27}$ The modulation frequency used has varied from 100 to $800 \mathrm{MHz}$ and the modulation depth decreased significantly with increase in the modulation frequency. If a primary focus of the fNIRS system design were to increase the modulation frequency an external modulation approach could be more appropriate. The external frequency modulation is usually provided by an electro-optic modulator incerted directly into the path of optical radiation in the fiber. The intensity of that radiation is changed by varying the current or voltage applied to the modulator. While such direct modulation increases the modulation depth at high frequencies, the capability of using the AC/DC ratio of intensities will be lost.

fNIRS systems with pulse modulated light sources are capable of providing simultaneous spatial and temporal information about light propagation in human tissue. ${ }^{28,29}$ Light sources used in those systems are usually pulsed laser diodes with pulse durations from $10 \mathrm{ps}$ to $10 \mathrm{fs}$ and repetition rates up to $100 \mathrm{MHz}$. The complexity of fNIRS systems with pulse modulated light sources, is determined by their weight, real estate required to accommodate them, and finally the cost which makes them currently impractical for airborne applications.

\section{B. Headgear}

Various designs of headgear for clinical use have been reported ${ }^{30-32}$ with the main focus on the functionality of the entire system. With an increasing interest in the development of an fNIRS system capable of operating on a human in motion, the efficiency of the headgear design started to attract attention. ${ }^{33}$

The main sources of power losses in the fNIRS system are coupling losses on interfaces. Owing to the fact that the human tissue is a highly scattering medium, most of the losses occur during ingress and egress of the light and it propagation through the human head. Thus, the signal to noise ratio can also be increased by improving the coupling of the injectors to the scalp. This issue has been addressed in a number of publications. ${ }^{34-37}$ Design of headgear plays an important role in the acceptance of fNIRS techniques by the clients including the pilot community and safety regulators. Ease of operation and comfort are also criteria used in the design. 
To evaluate the use of gradient index (GRIN) lenses to improve signal injection and collection, we considered two configurations. Gray matter phantoms were used to mimic the absorption and scattering properties of human brain tissue. Also, the light at two wavelengths, 690 and $830 \mathrm{~nm}$, was brought to the injectors via commercially available multimode fiber mode fiber with $50 \mu \mathrm{m}$ core diameter. Furthermore, the GRIN lenses were used as collectors to gather light scattered in the phantom.

Figure 2 shows the first two potential designs of optical injectors. In these two cases, as shown in the figure, the fibers are placed on the air-phantom interface and GRIN lenses are positioned in the plane normal to that interface. In Fig. 2a the injector is a right angle prism (RA Prism) which couples the light into the phantom by changing the direction of the light propagation by $90^{\circ}$. Such configuration permits continuing spreading of light after it exists the fiber and penetrates a wider but shallower area of the phantom. The injector shown in Fig. $2 \mathrm{~b}$ is a combination of a right angle prism and GRIN lens. The incorporation of a GRIN lens provides collimation of the light and a deeper penetration within the phantom.

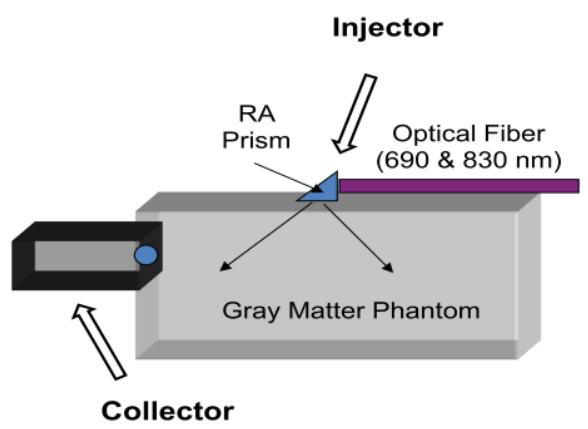

a)

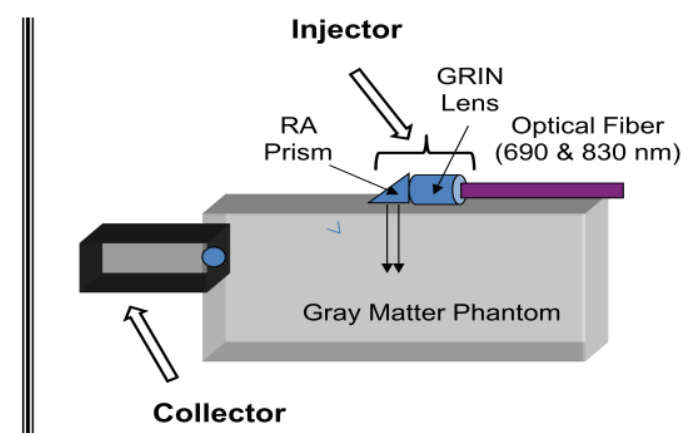

b)

Figure 2. Examples of optical injector configurations:

a) Injector with right angle prism;

b) Injector with right angle prism and GRIN lens.

In the third configuration shown in Fig. 3 the injector is a GRIN lens coupled to the fiber and the collector is also a GRIN lens and they both are facing the same phantom-air interface. A slight inclination of both injector and collector should enhance the directional coupling of light passing through the phantom material. However, this must be balanced against the subject's comfort.

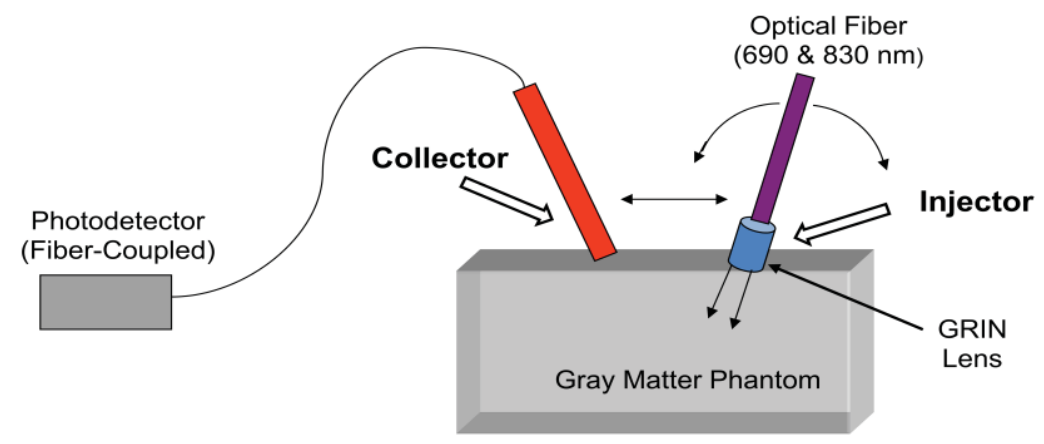

Figure 3. Example of the injector - collector configuration with GRIN lenses and mutual inclination

While other configurations of injectors are potentially available, the GRIN lens approach has shown to be the most promising of those considered. Most designs and numerical analyses of the GRIN lens-based probes have been done for a single wavelength operation. ${ }^{38,39}$ In fNIRS two wavelengths are being used and effects of GRIN lens on optical beams at those wavelengths are different. Figure 4 shows calculated spot diagrams of optical beams at 
two wavelengths, $690 \mathrm{~nm}$ (blue dots) and and $830 \mathrm{~nm}$ (red dots) after passing through a GRIN lens. The GRIN lens focuses the $690 \mathrm{~nm}$ wavelength beam at a distance of $7 \mathrm{~mm}$ from the exit aperture. The figure also shows clearly spreading of the beam at $830 \mathrm{~nm}$ wavelength.

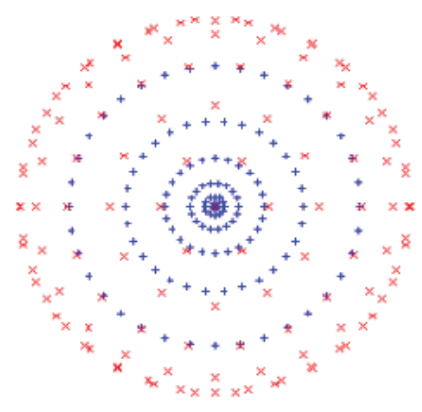

Figure 4. Calculated spot diagrams of optical beams at $690 \mathrm{~nm}$ (blue dots) and $830 \mathrm{~nm}$ (red dots) after passing through a GRIN lens

Thus, owing to the fact that fNIRS employs two wavelengths, better GRIN lens designs are needed to improve the coupling efficiency of the light into the human tissue. Furthemore, tests need to be performed on human volunteers in order to assess this light injector design's effectiveness at penetrating the cerebral-spinal fluid barrier and achieving cleaner scattered signals from the brain's gray matter.

\section{Signal Processing}

Minuaturization of fNIRS instrumentation involves development of compact modulation schemes to drive two light sources in a timely prescribed manner. Figure 5 demonstrates a laboratory prototype of electronic setup that permits multiplexing of modulated optical signals generated by two lasers at $690 \mathrm{nad} 830 \mathrm{~nm}$.

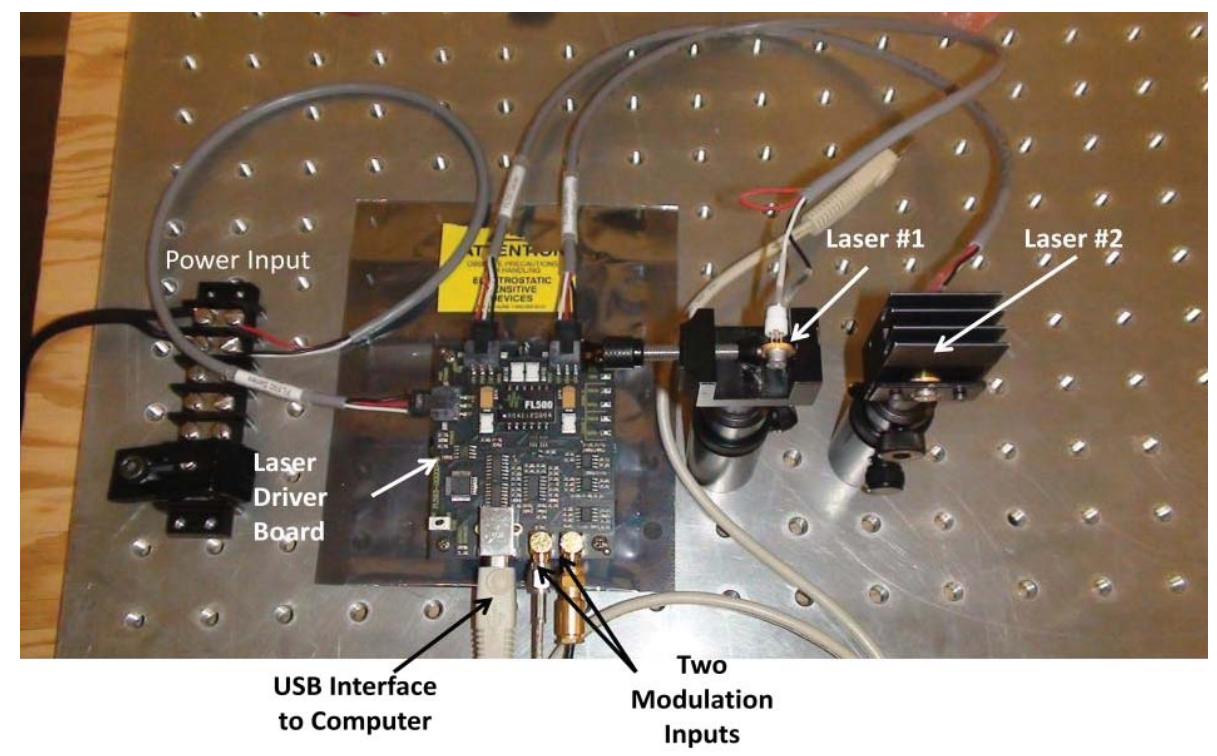

Figure 5. Laboratory electronic setup to multiplex two modulated optical signals generated by two lasers

The fNIRS systems usually involve headgears with multiple light injectors and collectors. The injectors and collectors are connected correspondingly to light sources and detectors via optical fibers. In airborne environments, in order to provide optical power to multiple injectors from light sources and deliver optical power from multiple collectors to photodetectors efficient optical power delivery multiplexing and demultiplexing techniques should be 
devise. Such techniques, in addition to channeling optical signals between the source and detector, should also minimize the number of connectorizing fibers. Thus, a properly designed optical signal delivery and collection configuration permits construction of a compact and efficient system with the optimum number of sources and detectors and significaltly reduced number of connecting fibers. Figures 6 and 7 show examples of pulsed multiplexing techniques that deliver light at two operational wavelengths, 690 and $830 \mathrm{~nm}$, to 4 optical injectors on one single headgear unit.

Figure 6 has two sets of light sources, either light emitting diodes or lasers. Each set has one light source emitting light at $690 \mathrm{~nm}$ and the other one at $830 \mathrm{~nm}$. Light sources in each set are pulse modulated in time with identical duty factor. In this figure the modulating frequencies for the light sources with similar wavelengths are shown to be identical. However, different modulating frequencies could be used as well to provide additional light source discrimination. The duty factors used in different sets may does not have to be identical.

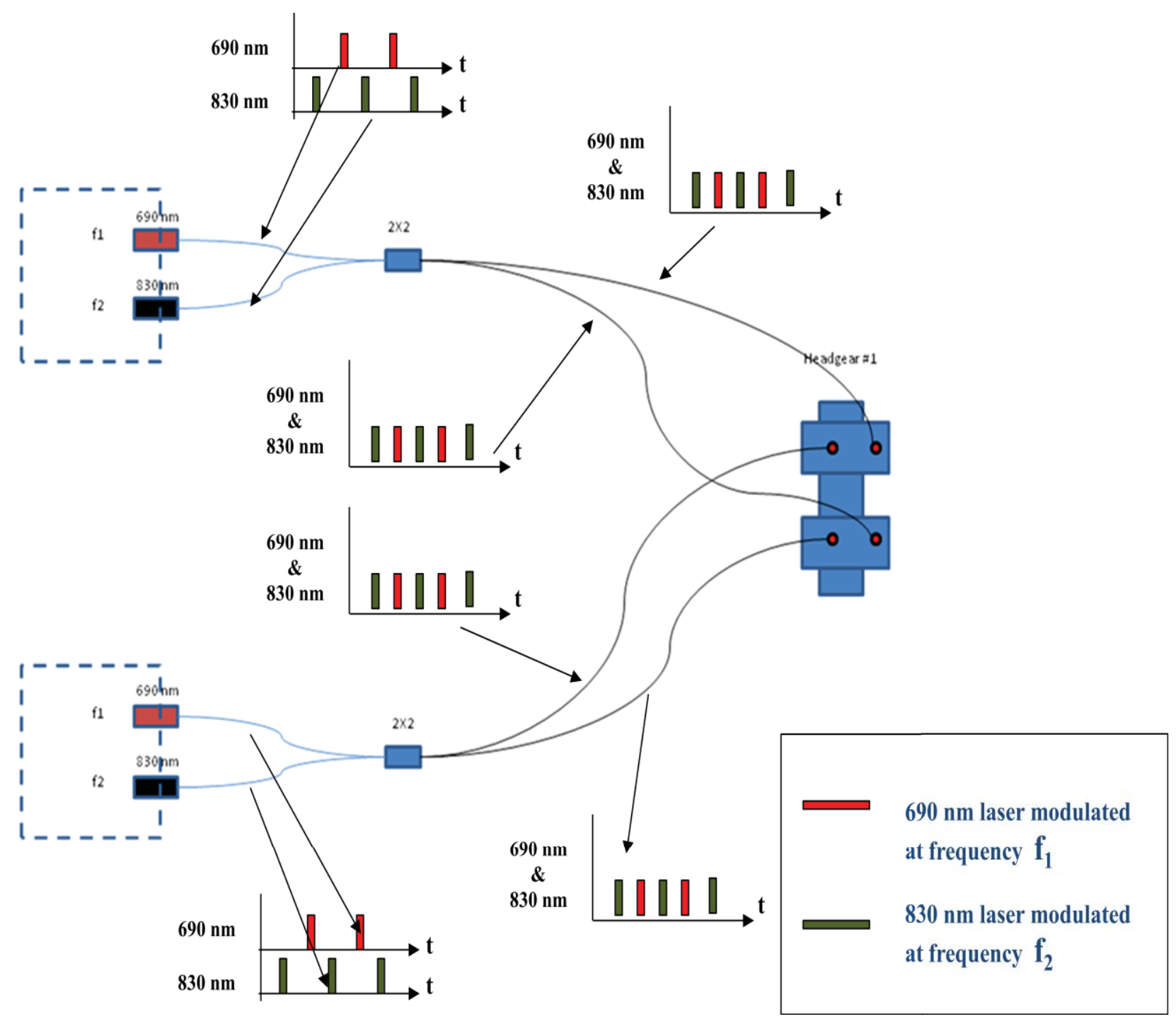

Figure 6. Example of a combined frequency-pulse multiplexed optical radiation delivery system

Figure 7 shows another configuration with multiplexed light sources. It differs from the one depicted in Fig. 6 by having additional asynchronous modulation of the light sources. 


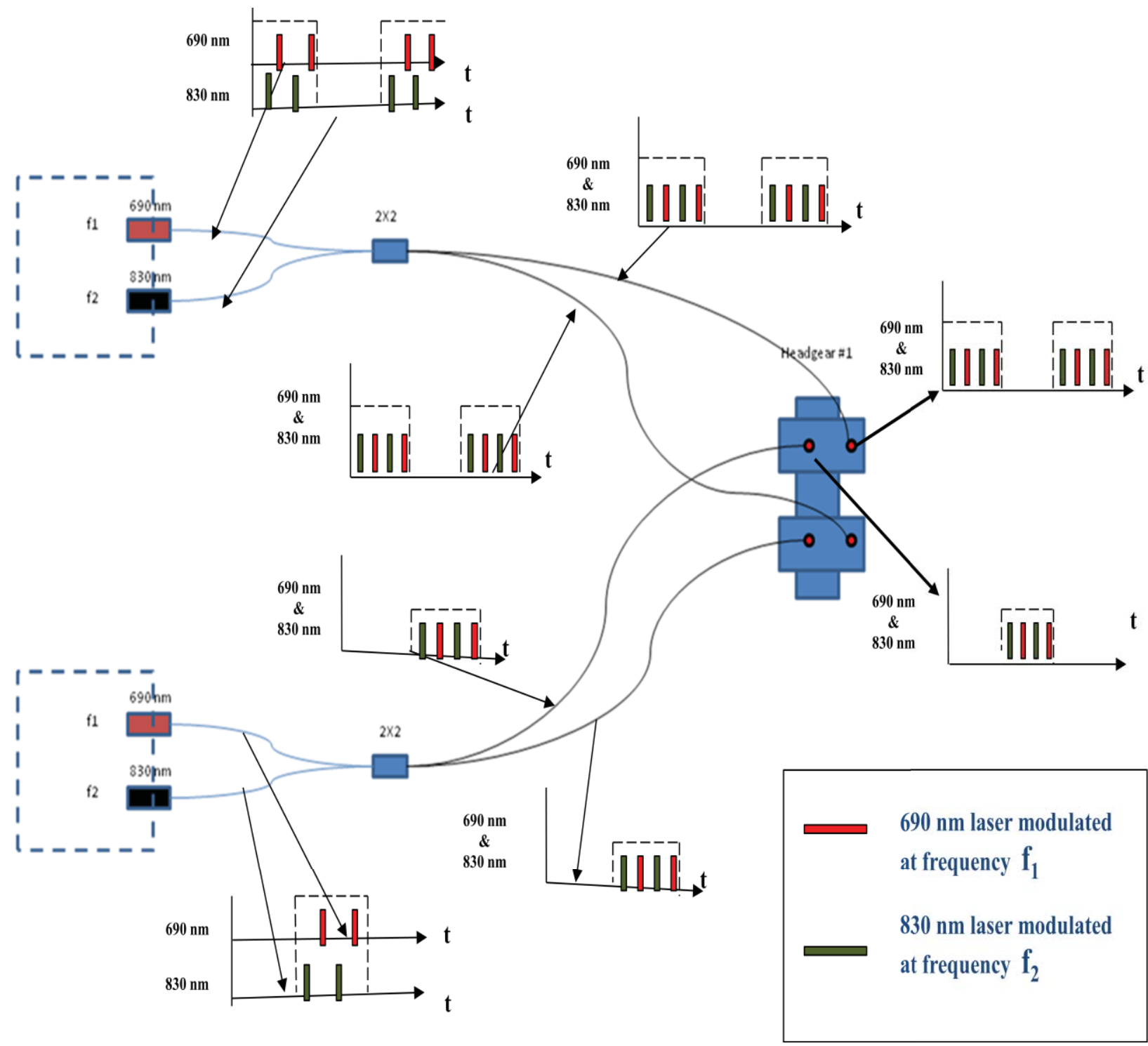

Figure 7. Example of a combined frequency-pulse multiplexed optical radiation delivery system with asynchronous modulation of the sources

\section{Numerical Analysis}

For continuous wave fNIRS, the relative concentration change in oxy- and deoxy-haemoglobin on a single channel are related to the optical intensities at two operating wavelengths measured from the corresponding instrument. ${ }^{40}$ Once relative changes in the concentration of the hemoglobin species have been determined, this data must be further post-processed to remove artifacts, filter noise, and ultimately classify the operator state. The use of fNIRS for state classification in an operational environment necessitates identification of data processing procedures which are demonstrably sufficient for delimiting between separable operator states, yet fast enough to operate under near real-time constraints. Solutions to the modified Beer-Lambert law can be computed by application of a single matrix-vector product. However, the filtering and classification procedures used to further process fNIRS data may be collectively more computationally expensive, depending on the exact post-processing procedures selected.

The ability to effectively distinguish between operator states using fNIRS is greatly enhanced by removing noise and artifacts from the data prior to conducting state classification. Many popular methods for filtering signals can be 
applied to fNIRS data to achieve this. Among them are wavelet filtering, blind source separation, and phaseadaptive Kalman filtering.

Wavelet filtering methods have recently been used to great effect to aid in the processing of fNIRS data. ${ }^{41-44}$ Among inherent features of discrete wavelet transforms that motivate their use for processing of fNIRS data are abilities to compress, denoise, and remove artifacts from single- and multidimensional digital signals. For example, the wavelet coefficients of digital signals tends to be sparse, allowing for efficient compression of data in wavelet transform domain. Wavelet transforms allow for the characterization of features within a signal to be examined at a variety of spatial or temporal scales, often referred to as Multi-Resolution Analysis (MRA). The wavelet coefficients of many digital signals tend to be uncorrelated in the transform domain, allowing for the de-correlation of independent signal features from background noise. And lastly, since each wavelet coefficient represents the content of the analyzed signal partially localized in both space (or time) and frequency, wavelet transforms provide simultaneous partial spatial and frequency localizations.

Since applications of fNIRS typically involve the use of multiple channels of input data, denoising and artifact removal could also be potentially achieved using blind source separation techniques. Blind source separation techniques are algorithms which seek to systematically identify and separate unknown signals given a collection of mixed signal and noise data, without a priori knowledge of the underlying mixing process. These methods typically operate by iteratively minimizing some mutual measure of statistical independence (such as sample correlation) between lists of given data, under the assumption of an overall linear mixing process.

Principal component analysis (PCA) and independent component analysis (ICA) are two popular methods of blind source separation and their applications to fNIRS for both offline and real time processing are well described in the literature. ${ }^{45-49}$

Phase-adaptive Kalman filtering is another technique that has been recently introduced. It is specific to frequency domain fNIRS instrumentation ${ }^{50}$ and involves the application of an adaptive Kalman filter to the computed values of hemoglobin. In the application of the technique the intensity of the Kalman filter applied to each channel is tuned according to the variability of the corresponding phase shift channel. The principal behind this procedure is that high variation in the phase shift channel is associated with poor coupling of the associated optrode to the skin. Such a measure, taken over a short frame of time, provides a strong indicator of motion artifacts and an overall poor signal-to-noise ratio within that same block of time. The method has a number of advantages over alternative filtering operations that have been described. It does not introduce ringing or overshooting artifacts at the endpoints of the data, as certain combinations of wavelet filters and boundary conditions may introduce while conducting wavelet filtering. ${ }^{51}$ In contrast to PCA or ICA, since this filtering is performed independently for each channel, it is applicable for cases where there are limited channels (or even a single channel) of interest.

\section{Discussion}

Currently used commercial fNIRS instrumentation is designed for hospitals and other clinical or laboratory environments. To make the fNIRS technology suitable for airborne applications, the conventional approaches should be reevaluated to make the instrumentation small, light, airworthy, and pilot-friendly.

The design of the entire system should be based on the minimum resources availability. That includes space and weight limitations as well as low power consumption. While a broad range of optical sources, detectors, and fiber optic components is available for operational wavelengths in the communication range, the wavelength range from 600 to $900 \mathrm{~nm}$ still lacks similar diversity. Thus, with a relatively limited vendor's base, configurations of the optical signal delivery and collection system should be designed to achieve the maximum signal-to-noise ratio and maximum efficiency.

The design of headgear should provide, in addition to functionality, comfort and safety during pilot's involvement in cockpit activities. The issue of comfort and client cooperation should be addressed by minimizing the number of fiber optic links connecting the headgear with signal processing units including optical sources and detectors. Furthermore, the design of the headgear itself should be user friendly and not restrictive. Multiplexing schemes should be developed to minimize the number of fiber optic cables connecting the headgear with the signal processing equipment without negative effects on the signal-to-noise ratio. Moreover, development of appropriate wireless configurations should be considered to remove any constrains on the movement of pilots in the cockpit.

Safety issues should also be considered in the process of selection of optical sources. While the current safety standards regarding minimum permissible exposure to optical radiation regulate the human skin exposure ${ }^{52}$, no such standards exist when the radiation is applied to human head. Effects of a long-term exposure of human scalp and brain tissue to high power highly focused optical radiation in 600 to $900 \mathrm{~nm}$ wavelength range with potentially deep penetration have not been fully studied. 
The software for real time data processing should be developed under an assumption of constrained computational resources specific to aviation data systems. Numerical models and software for such conditions are being currently developed ${ }^{52,53}$ and their adaptation to cockpit applications of fNIRS would further enhance the technology.

Finally, the cockpit environment requires a strict certification for any equipment brought to it. Therefore, the fNIRS instrumentation developed for airborne environments should have low electromagnetic emission and minimum electromagnetic interference with the already existing equipment.

\section{Conclusion}

While the fNIRS has found wide applications in the medical field the use of the technology on human subjects in airborne environments is practically nonexistent. One of the issues detrimental to the technology incertion into such environments is a lack of suitable instrumentation packages and associated software.

The paper clearly identifies major areas in the development and implementation of instrumentation and software related elements for airborne fNIRS systems. It also suggests a path for the technology maturation as articulated in the discussion section. As fNIRS is shown to be useful for detecting cognitive state and improving aviation safety, the technical maturation of these elements specifically toward use in airborne environments will become more warranted.

\section{Acknowledgments}

The work has been supported by the Vehicle System Safety Technologies (VSST) Project of NASA Aviation Safety Program. The authors wish to express gratitude to Mr. Christopher Blasio, NASA GRC Radiation and Laser Safety Officer for useful advice, and Mr. Daniel Gotti of National Center for Space Exploration Research for assistance with design and construction of the headgear.

\section{References}

${ }^{1}$ De Nuovo, A., G., Cannavo, R., B., and Di Nuovo, S., “An Agent-based Infrastructure for Monitoring Aviation Pilot's Situation Awareness," IEEE Conference Publication, 2011 IEEE Symposium on Intelligent Agents (IA), 2011, pp 1-7.

${ }^{2}$ Kikukawa, A., Kobayaashi, A., and Miyamoto, Y., "Monitoring of Pre-frontal Oxygen Status in Helicopter Pilot Using Near-Infrared Spectrophotometers," Dynamic Medicine 2008, 7:10 [online journal], URL: http://www.dynamicmed.com/content/7/1/10 [cited 5 July 2013].

${ }^{3}$ Mumaw, R., J., Sarter, N., B., and Wickens, C. D., “Analysis of Pilot's Monitoring and Performance on an Automated Flight Deck," Presentation, 11th International Symposium on Aviation Psychology, The Ohio State University, Columbus , OH, 2001, URL: http://www.aviation.illinois.edu/avimain/papers/research/pub pdfs/isap/starter.pdf [cited 5 July, 2013].

${ }^{4}$ Colvin, K., Dodhia, R. M., Belcher, S., and Dismukes, K., "Scanning for Visual Traffic: An Eye Tracking Study," Presentation, 11th International Symposium on Aviation Psychology, The Ohio State University, Columbus , OH, 2001, URL: http://hsi.arc.nasa.gov/flightcognition/Publications/Scanning.pdf $\quad$ [cited $7 \quad$ June 2013] and URL: http://humanfactors.arc.nasa.gov/flightcognition/Publications/Scanning.pdf [cited 14 June 2013].

${ }^{5}$ Hawrysz, D. and Sevick-Muraca, E., "Developments Toward Diagnostic Breast Cancer Imaging Using Near-Infrared Optical Measurements and Fluorescent Contrast Agents," Neoplasia, Vol. 2, No. 5, 2000, pp. 388-417.

${ }^{6}$ McBride, T. O., "Spectroscopic Reconstructed Near Infrared Tomographic Imaging for Breast Cancer Diagnosis," Ph.D. Dissertation, Thayer School of Engineering, Darthmouth College, Hanover, NH, 2002.

${ }^{7}$ Gervain, J., Mehler, J., Werker, J. F., Nelson, C. A., Csibra, G., Lloyd-Fox, S., Shukla, M., and Aslin, R. N., "Near-Infrared Spectroscopy: A Report from the McDonnel Infant Methodology Consortium," Developmental Cognitive Neuroscience, Vol. 1, 2011, pp. 22-46.

${ }^{8}$ Taga, G., Asakawa, K., Maki, A., Konishi, Y., and Koizumi, H., "Brain Imaging in Awake Infants by Near-Infrared Optical Topography," Proceedings of the National Academy of Science of the United States of America, Vol. 19, No. 19, 2003, pp. 10722-10727.

${ }^{9}$ Orihuela-Espina, F., Leff, D. R., James, D. R. C., Darzi, A. W., and Yang, G., Z., "Quality Control and Assurance in Functional Near Infrared Spectroscopy (fNIRS) Experimentation,” Phys. Med. Biol., Vol. 55, 2010, pp. 3701-3724.

${ }^{10}$ Lloyd-Fox, S., Blasi, A., and Elwell, C. E., "Illuminating the Developing Brain: The Past, Present and Future of Functional Near Infrared Spectroscopy," Neuroscience and Biobehavoral Reviews, Vol. 34, 2010, pp. 269-284.

${ }^{11}$ Murkin, J. M. and Arango, M., "Near-infrared Spectroscopy as an Index of Brain and Tissue Oxygenation," British Journal of Anaesthesia, Vol. 103 (Suppl. I), 2009, pp. i3-i13.

${ }^{12}$ Yodh, A. G. and Boas, D. A., "Functional Imaging with Diffusing Light," Biomedical Photonics Handbook, edited by T.Vo-Dinh, Chapter 21, CRC Press, Boca Raton, Florida, 2003, pp. from 21-1 to 21-45.

${ }^{13}$ Matthews, F., Pearlmutter, B. A., Ward, T. E., Soraghan, C., and Markham, C., "Hemodynamics of Brain-Computer Interfaces," IEEE Signal Processing Magazine, Vol. 25, No. 1, 2008, pp.87-94. 
${ }^{14}$ Bunce, S. C., Izzetoglu, M., Izzetoglu, K., Onaral, B., and Pourrezaei, K., “Functional Near-Infrared Spectroscopy,” IEEE Engineering in Medicine and Biology Magazine, Vol. 25, No. 1, 2006, pp. 54-62.

${ }^{15}$ Boas, D. A., Gaudette, T., Strangman, G., Chang, X., Marota, J. J. A., and Mandeville, J. B., "The Accuracy of Near Infrared Spectroscopy and Imaging during Focal Changes in Cerebral Hemodynamics," NeuroImage, Vol. 13, 2001 , pp. 76-90.

${ }^{16}$ Izzetoglu, M., Bunce, S. C., Izzetoglu, K., Onaral, B., and Pourrezaei, K., "Functional Brain Imaging Using Near-Infrared Technology," IEEE Engineering in Medicine and Biology Magazine, July/August 2007, pp. 38-46.

17 Yamashita, Y., Maki, A., and Koizumi, H., "Wavelength Dependence of the Precision of Noninvasive Optical Measurements of Oxy-, Deoxy, and Total-Hemoglobin Concentration,” Med. Phys., Vol. 28, No. 6, 2001, pp. 1108-1114.

${ }^{18}$ Sato, H., Kiguchi, M., Kawaguchi, F., and Maki, A., "Practicality of Wavelength Selection to Improve Signal-to-Noise Ratio in Near-Infrared Spectroscopy," NeuroImage, Vol. 21, 2004, pp. 1554-1562.

${ }^{19}$ Coyle, S. M., Ward, T. E., and Markham, C. M., "Brain-Computer Interface Using a Simplified Functional Near-Infrared Spectroscopy System,” J. Neural Eng., Vol. 4, 2007, pp. 219-226.

${ }^{20}$ Chang, G.-C., Wang, K.-J., Hsu, C.-H., and Chen, J.-J. J., "Development of Functional Near Infrared Spectroscopy System for Assessing Cerebral Hemodynamics of Rats with Ischemic Stroke,” J. Med. Biol. Eng., Vol.27, No. 4, 2007, pp. $207-213$.

${ }^{21}$ Dehaes, M ., Grant, P. E., Sliva, D., D., Roche-Labarbe, N., Pienaar, R., Boas, D., A., Franceschini, M. A., and Selb, J., "Assessment of the Frequency-Domain Multi-Distance method to Evaluate the Brain properties: Monte Carlo Simulation from Neonate to Adult," Biomed. Opt. Express, Vol. 2, No. 3. 2011, pp. 552-567.

${ }^{22}$ Huppert, T. J., Diamond, S. G., Franceschini, M. A., and Boas, D. A., "HomER: A review of Time-Series Analysis Methods for Near-Infrared Spectroscopy of the Brain,” Appl. Opt.,Vol. 48, No. 10, 2009, pp. D280- D298.

${ }^{23}$ Kurth, C. D. and Thayer, W. S., “A A Miltiwavelength Frequency-Domain Near-Infrared Cerebral Oximeter,” Phys. Med. Biol., Vol. 44, 1999, pp. 727-740.

${ }^{24}$ Pogue, B. W., Testorf, M., McBride, T., Osterberg, U., and Paulsen, K., "Instrumentation and Design of a FrequencyDomain Diffuse Optical Tomography Imager for Breast Cancer Detection," Optics Express, Vo1. 1, No. 13, 1997, pp. 391 -403.

${ }^{25}$ Fantini, S. and Franceschini, M. A., "Frequency-domain techniques for tissue spectroscopy and imaging," URL: http://ase.tufts.edu/biomedical/research/fantini/publications/NIRS experimental/6 Chapter 7.PDF

${ }^{26}$ Chipchase, J., Kirkby, D., Peebles, D., and Cope, M., "Cerebral Hemoglobin Concentration and Oxygen Saturation Measured by Intensity Modulated Optical Spectroscopy in Human Fetus during Labor,” J. Perinat. Med., Vol. 30, 2002, pp. 502509.

${ }^{27}$ Toronov, V., D’Amico, E., Hueber, D., Gratton, E., Barbieri, B., and Webb. A., “Optimization of Signal-to-Noise Ratio of Frequency-Domain Instrumentation for Near-Infrared Spectro-Imaging of Human Brain," Optics Express, Vol. 11, No. 21, 2003, pp. 2717-2729.

${ }^{28}$ Contini, D., Torricelli, A., Pifferi, A., Spinelli, L., Paglia, F., and Cubeddu, R., "Multichannel Time-Resolved System for Functional Near Infrared Spectroscopy," Optics Express, Vol. 4, No. 12, 2006, 5418-5432.

${ }^{29}$ Torricelli, A., Contini, D., Pifferi, A., Caffini, M., Re, R., Zucchelli, L., and Spinelli, L., "Time Domain Functional NIRS Imaging for Human Brain Mapping," NeuroImage, 2013, URL: http://dx.doi.org/10.1016/j.neuroimage.2013.05.106 [cited 10 July 2013].

${ }^{30}$ Coyle, S., Markham, C., Lanigan, W., and Ward, T., "A Mechanical Mounting System for Functional Near-Infrared Spectroscopy Brain Imaging Studies,” Proc. SPIE 5826, Opto-Ireland 2005: Optical Sensing and Spectroscopy, 2005, pp. 618627, doi:10.1117/12.604823.

${ }^{31}$ Khan, B., Widley, C., Francis, R., Tian, F., Delgado, M., R., Liu, H., MacFarlane, D., and Alexandrakis, G., "Improving Optical Contact and Functional Near-Infrared Brain Spectroscopy and Imaging with Brush Optrodes," Biomedical Optics Express, Vol. 3, No. 5, 2012, pp. 878-896.

${ }^{32}$ Giacometti, P. and Diamond, S., G., "Compliant head Probe for Positioning Electroencephalography Electrodes and NearInfrared Spectroscopy Optrodes," J. Biomed. Optics, Vol. 18, No. 2, 2013, 027005.

${ }^{33}$ Zhang, Q., Yan, X., and Strangman, G., E., "Development of Motion Resistant Instrumentation for Ambulatory NearInfrared Spectroscopy,” J. Biomed, Optics, Vol. 16, No. 8, 2011, 097008.

${ }^{34}$ Mackey, J. R., Adamovsky, Tin, P., Floyd, B. M., and Harrivel, A. R., "Improved Light Injection and Detection Methods for fNIRS Headgear for use in Avionics and Astronautics," Abstract Book, Functional Near Infrared Spectroscopy Conference, University College London, London, UK, $26^{\text {th }}-28$ October 2012, p. 73, URL: http://www.fnirs.org/fnirs2012/assets/fNIRS2012abstract-book.pdf [cited 17 July 2013].

${ }^{35}$ Harrivel, A. R., Hylton, A. G., and Hearn, T. A., "Best Practices for the Application of Functional Near Infrared Spectroscopy to Operator State Sensing," NASA TM-2012-217615, 2012.

${ }^{36}$ Harrivel, A. R., McKay, T. L., Hylton, A. G., King, J. K., Latorella, K. A., Peltier, S. J., and Noll, D. C., "Toward Improved Headgear for Monitoring with Functional Near Infrared Spectroscopy," NeuroImage, Vol. 47, Supplement 1, 2009 , p. S141.

${ }^{37}$ Coyle, S. M, Ward, T. E., and Markham, C. M., "Brain-Computer Interface Using a Simplified Near-Infrared Spectrometry System," J. Neural Eng., Vol. 4, 2007, pp.219-226.

${ }^{38}$ Jung, W., Benalcazar, W., Ahmad, A., Sharma, U., Tu, H., and Boppart, S. A.," Numerical Analysis of Gradient Index Lens-based Optical Coherence Tomography Imaging Probes," J. Biomed. Opt., Vol. 15, No. 6, 2010, 06627.

${ }^{39}$ Evans, J., "Simple Forms for Equations of Rays in Gradient-Index Lenses," Am. J. Phys., Vol. 58, No. 8, 1990, pp. 773778. 
${ }^{40}$ Abdelnour, A. F. and Huppert, T., "Real-time Imaging of Human Brain Function by Near-Infrared Spectroscopy Using an Adaptive General Linear Model," Neuroimage, Vol. 6, No. 1, 2009, pp. 133-143.

${ }^{41}$ Khoa, T. Q. D. and Nakagawa, M., "Functional Near Infrared Spectroscope for Cognition Brain Tasks by Wavelets Analysis and Neural Networks," Int. J. Biol. Med. Sci., Vol. 1, No. 1, 2008, pp. 28-33.

${ }^{42}$ Li, Z., Wand, Y., Li, Y., Wang, Y., Li, J., and Zhang, L., "Wavelet Analysis of Cerebral Oxygenation Signal Measured by Near Infrared Spectroscopy in Subjects with Cerebral Infarction," Microvascular Research, Vol. 80, No. 2, 2010, pp.142-147.

${ }^{43}$ Cui, X., Bryant, D. M., and Reiss, A. L., "NIRS-Based Hyperscanning Reveals Increased Interpersonal Coherence in Superior Frontal Cortex during Cooperation," Neuroimage, Vol. 59, No. 3, 2012, pp. 2430-2437.

${ }^{44}$ Molavi, B. and Guy A. Dumont, G. A., "Wavelet-Based Motion Artifact Removal for Functional Near-Infrared Spectroscopy," Physiological Measurement, Vol. 33, No. 2, 2012, pp 259-271.

${ }^{45}$ Virtanen, J., Noponen, T., and Meriläinen, P., "Comparison of Principal and Independent Component Analysis in Removing Extracerebral Interference from Near-Infrared Spectroscopy Signals," J. Biomed. Opt., Vol. 14, No. 5, $2009,054032$. URL: http://dx.doi.org/10.1117/1.3253323.

${ }^{46}$ Tak, S. and Ye, J.C., "Statistical Analysis of fNIRS Data: A Comprehensive Review." Neuroimage, 2013, URL: http://dx.doi.org/10.1016/j.neuroimage.2013.06.016 [cited 16 July 2013].

${ }^{47}$ Zhang, H., Zhang, Y.-J, Lu, C.-M., Ma, S.-Y., Zang, Y.-F., and Zhu, C.-Z., "Functional Connectivity as Revealed by Independent Component Analysis of Resting-State fNIRS Measurements," Neuroimage, Vol. 51, No.3, 2010 , pp. $1150-1161$.

${ }^{48}$ Ayaz, H., Izzetoglu, M., Shewokis, P. A., and Onaral, B., "Sliding-Window Motion Artifact Rejection for Functional NearInfrared Spectroscopy." Proceedings of the $32^{\text {nd }}$ Annual International Conference of the IEEE EMBS, Buenos Aires, Argentina, August 31-September 4, 2010, pp. 6567-6570, URL:

http://www.biomed.drexel.edu/fnir/conquer/fNIR_Publications_files/C\%20Ayaz\%20-2010\%20-\%20EMBC2010.pdf [cited 16 July 2013].

${ }^{49}$ Kohno, S., Miyai, I., Seiyama, A., Oda, I., Ishikawa, A, Tsuneishi, S., Ameta, T., and Shimizu, K., "Removal of the Skin Blood Flow Artifact in Functional Near-Infrared Spectroscopic Imaging Data through Independent Component Analysis." J. Biomed. Optics, Vol. 12, No. 6, 2007, 062111-062111.

${ }^{50}$ Harrivel, A. and Hearn, T., "Functional Near Infrared Spectroscopy: Watching the Brain in Flight", URL: http://ntrs.nasa.gov/archive/nasa/casi.ntrs.nasa.gov/20120015039_2012015346.pdf [cited 17 July 2013].

${ }^{51}$ Unser, M., "A practical guide to the implementation of the wavelet transform," Wavelets in Medicine and Biology, edited by A. Aldroubi and M. Unser, CRC Press, 1996, pp. 37-73.

${ }^{52}$ Laser Safety, URL: http://en.wikipedia.org/wiki/Laser_safety [cited 10 July 2013].

${ }^{53}$ Memik, S. O., Katsaggelos, A. K., and Sarrafzadeh, M., "Analysis and FPGA Implementation of Image Restoration under Resources Constrains," IEEE Transactions on Computers, Vol. 52, No. 3, 2003, pp.1-10.

${ }^{54}$ Johnston, C. T., Gribbon, K. T., and Bailey, D. G., "Implementating Image Processing Algorithms on FPGAs," URL: http://seat.massey.ac.nz/research/centres/SPRG/pdfs/2004_ENZCON_118.pdf [cited 17 July 2013]. 